\title{
Der Kandidat / die Kandidatin - das gar nicht mehr so unbekannte Wesen ${ }^{*}$
}

\author{
Philip Manow und Peter Flemming
}

Wir wissen mittlerweile recht viel über die Abgeordneten des Deutschen Bundestages, über ihr Repräsentationsverständnis ${ }^{1}$, ihre parlamentarischen Strategien ${ }^{2}$, ihre Wiederwahlchancen $^{3}$, ihr Wahlkampfverhalten ${ }^{4}$ etc. Systematische Informationen über die Wahlbewerber für den Bundestag sind jedoch bis heute spärlich. ${ }^{5}$ Dies schließt auch Unwissen über das Vorund Nachleben der Abgeordneten ein: Wie häufig kandidieren Mitglieder des Bundestags, bevor ihnen der Einzug in das Parlament erstmalig gelingt? Wie häufig kandidieren sie noch erfolglos nach ihrer letzten Mitgliedschaft im Bundestag? Wie genau scheiden Abgeordnete aus dem Bundestag aus - durch Abwahl oder durch Nicht-Wiedernominierung? Dies sind einfache, aber bislang weitgehend unbeantwortete Fragen ${ }^{6}-$ Fragen von grundsätzlicher

Wir danken Holger Döring und Pawel Szczerbak für ihre Hilfe bei der Datengenerierung. Wir danken zudem Ulrich Kohler, André Kaiser und Valentin Schröder für hilfreiche Kommentare zu früheren Versionen dieses Papiers.

1 Vgl. Werner Patzelt, Abgeordnete und Repräsentation. Amtsverständnis und Wahlkreisarbeit, Passau 1993; ders., German MPs and their Roles, in: Journal of Legislative Studies, 3. Jg. (1997), H. 1, S. 55 - 78; Thomas Zittel, Legislators and their Representational Roles: Strategic Choice or Habits of the Heart?, in: Magnus Blomgren / Olivier Rozenberg (Hrsg.), Parliamentary Roles in Modern Legislatures, London / New York, S. 101 - 120; Carl Christoph Schweitzer, Der Abgeordnete im parlamentarischen Regierungssystem der Bundesrepublik, Opladen 1979; HansDieter Klingelmann / Wolfgang Wessels, The Consequences of Germany's Mixed-Member System: Personalization at the Grassroots?, in: Matthew S. Shugart / Martin P. Wattenberg (Hrsg.), Mixed Member Electoral Systems. The Best of Two Worlds?, Oxford 2001, S. 279 - 296.

2 Vgl. Werner Patzelt, German MPs and their Roles, a.a.O. (Fn. 1), S. 1; ders., What Can an Individual MP do in German Parliamentary Politics?, in: Journal of Legislative Studies, 5. Jg. (1999), H. 3, S. 23 - 52; Thomas Stratmann / Martin Baur, Plurality Rule, Proportional Representation, and the German Bundestag: How Incentives to Pork-Barrel Differ Across Electoral Systems, in: American Journal of Political Science, 46. Jg. (2002), H. 3, S. 506 - 514; Thomas D. Lancaster 1 William D. Patterson, Comparative Pork Barrel Politics Perceptions from the West-German-Bundestag, in: Comparative Political Studies, 22. Jg (1990), H. 4, S. 458 - 477; Ulrich Sieberer, Behavioral Consequences of Mixed Electoral Systems: Deviating Voting Behavior of District and List MPs in the German Bundestag, in: Electoral Studies, 29. Jg. (2010), H. 3, S. $484-496$.

3 Vgl. Philip Manow, Wiederwahlchancen im deutschen System der personalisierten Verhältniswahl - eine empirische Untersuchung der sechzehn Bundestagswahlen 1949-2005, in: ZPol, 18. Jg. (2008), H. 2, S. $147-166$.

4 Vgl. Thomas Zittel/ Thomas Gschwend, Individualisierte Wahlkämpfe im Wahlkreis. Eine Analyse am Beispiel des Bundestagswahlkampfes von 2005, in: PVS, 48. Jg. (2007), H. 2, S. $293-321$.

5 Vgl. Suzanne S. Schüttemeyer / Roland Sturm, Der Kandidat - das (fast) unbekannte Wesen. Befunde und Überlegungen zur Aufstellung der Bewerber zum Deutschen Bundestags, in: ZParl, 36. Jg. (2005), H. 3, S. 539 - 553; Bernhard Weßels, Germany, in: Pippa Norris (Hrsg.), Passages to Power, New York 1997, S. 76 - 97; Geoffrey Roberts, The German Federal Republic: The Two-lane Route to Bonn, in: Michael Gallagher / Mitchell Marsh, Candidate Selection in Comparative Perspective: The Secret Garden of Politics, London 1988, S. $94-118$.

6 Vgl. Michael Edinger / Bertram Schwarz, Leben nach dem Mandat. Eine Studie zu ehemaligen Abgeordneten, in: SFB 580 - Gesellschaftliche Entwicklung nach dem Systemzusammenbruch (Hrsg.), H. 35, Jena 2009. 
Bedeutung, wenn man die Effektivität des Instruments der demokratischen Wahl abschätzen will. ${ }^{7}$ Es geht um fundamentale demokratische Mechanismen: Wer sanktioniert - der Wähler oder die Parteien? In welchem Ausmaß bietet schon die Nominierung durch eine Partei die leidlich sichere Aussicht auf eine tatsächlich erfolgreiche Wahl und Wiederwahl?

Es geht auch um grundlegende Fragen demokratischer Repräsentation: Aus welchem Kandidatenpool können Wähler auswählen beziehungsweise resultieren Defizite „deskriptiver Repräsentation " 8 aus der Kandidatennominierung der Parteien oder aus dem Wahlverhalten der Wähler - oder aus dem Zusammenspiel beider? So ist etwa in Bezug auf die Präsenz von Frauen im Parlament danach zu fragen, wo genau Selektionsmechanismen möglicherweise greifen: bereits bei der Nominierung oder erst bei der Wahl?? Und wenn schon bei der Nominierung, wird es zentral zu wissen, wie genau Frauen kandidieren: als Listen-, Direkt- oder Doppelkandidaten, auf welchen Listenplätzen und in welchen Wahlkreisen? Und gibt es in dieser Hinsicht Unterschiede zwischen den Parteien?

Zur Beantwortung dieser Fragen wünscht man sich idealerweise nach Parteien differenzierende Daten zu Abgeordneten und Kandidaten einschließlich der Information zum Modus der Kandidatur (Wahlkreis-, Listen-, Doppelkandidatur) mit Angaben zu den Listenplätzen und Wahlkreisen und Wahlkreisergebnissen. Diese Datengrundlage fehlte jedoch bislang für die Bundesrepublik Deutschland. Dabei liegen die betreffenden Informationen natürlich grundsätzlich vor: In den vom Statistischen Bundesamt zu jeder Bundestagswahl herausgegebenen Sonderheften „Die Wahlbewerber zur Wahl des ... Deutschen Bundestages" finden sich beispielsweise alle zu einer Wahl antretenden Personen verzeichnet, lange Zeit sogar mit Berufs- und genauer Adressenangabe. Die Stimmenergebnisse auf Wahlkreisebene liegen ebenfalls vor. Will man also mehr über grundlegende Mechanismen der Repräsentation wissen, wäre ein erster wichtiger Schritt, die bereits vorhandenen Informationen zu erheben, digital lesbar zu machen und miteinander zu verbinden. Zum Teil kann für dieses Vorhaben auf wertvolle Vorarbeiten zurückgegriffen werden. ${ }^{10}$

In einem an den Universitäten Konstanz, Heidelberg und nun Bremen angesiedeltem Datenprojekt haben wir die Erstellung eines solchen umfassenden Datensatzes betrieben.

7 Vgl. G. Bingham Powell, Elections as Instruments of Democracy. Majoritarian and Proportional Visions, New Haven / London 2000.

8 Anne Phillips, The Politics of Presence. The Political Representation of Gender, Ethnicity, and Race, Oxford 1995.

9 Vgl. Wilma Rule, Electoral Systems, Contextual Factors and Women's Opportunity for Election to Parliament in Twenty-Three Democracies, in: Western Political Quarterly, 40. Jg. (1987), H. 3, S. 477 - 498; Lena Wängnerud, Women in Parliaments: Descriptive and Substantive Representation, in: Annual Reviews of Political Science, 12. Jg. (2009), S. 51 - 69; Mercedes Mateo Diaz, Representing Women: Female Legislators in West European Parliaments, London 2008.

10 Vgl. Martin Schumacher, M.d.B. - Volksvertretung im Wiederaufbau. Bundestagskandidaten und die Mitglieder der westzonalen Vorparlamente. Eine biographische Dokumentation, Düsseldorf 2000; Michael F. Feldkamp, Deutscher Bundestag 1987 bis 2005: Parlaments- und Wahlstatistik, in: ZParl, 37. Jg. (2006), H. 1, S. 3 - 19; ders., Datenhandbuch zur Geschichte des Deutschen Bundestages 1994 - 2003, Ergänzungsband, Baden-Baden 2005; Peter Schindler, Datenhandbuch zur Geschichte des Deutschen Bundestages 1949 bis 1982, Baden-Baden 1984; ders., Datenhandbuch zur Geschichte des Deutschen Bundestages, Baden-Baden 1998; ders., Datenhandbuch zur Geschichte des Deutschen Bundestages 1949 bis 1999, Baden-Baden 1999; Rudolf Vierhaus I Ludolf Herbst, Biographisches Handbuch der Mitglieder des Deutschen Bundestages 1949 - 2003, München 2003. 
Aus verschiedenen Quellen wurden alle jemals an einer Bundestagswahl (von 1949 bis 2009) als Kandidaten teilnehmende Personen erfasst - und damit als Untergruppe auch alle jemals in den Bundestag Gewählten. Im Folgenden soll dieser Datensatz zur Klärung der oben aufgeführten grundsätzlichen Fragen zur Funktionsweise demokratischer Repräsentation genutzt und ein Ausblick auf weitere Forschungsfragen gegeben werden, die sich mithilfe unseres Kandidaten- und Abgeordnetendatensatzes (KAD-BT) werden beantworten lassen. ${ }^{11}$ Der Prozess der Datengenerierung, -bereinigung und -validierung sowie der Datensatz selbst werden ausführlich im Anhang zu diesem Beitrag beschrieben.

\section{Mechanismen demokratischer Repräsentation in der Bundesrepublik Deutschland}

Eine erste wesentliche Frage lautet, ob man bei Kandidaten und Abgeordneten von zwei verschiedenen Populationen sprechen kann. Einiges scheint tatsächlich dafür zu sprechen. So hatten von den insgesamt 3.581 Abgeordneten ganze 79,6 Prozent vor ihrem ersten Einzug in den Bundestag noch nie kandidiert. Ihnen gelang es also im ersten Anlauf, einen Sitz im Parlament zu erringen. Es zeigt sich überdies, dass wiederum von allen Abgeordneten 76,4 Prozent nach ihrer ersten Wahlperiode nie wieder kandidiert haben, ohne nicht auch gewählt worden zu sein. Es haben also nur etwa 20 Prozent aller Abgeordneten vor sowie 24 Prozent nach ihrem ersten Bundestagseinzug mindestens einmal erfolglos kandidiert. Differenziert man diese Betrachtung nach Parteien wird schnell ersichtlich, dass es vor allem SPD und CDU/CSU sind, die für diese hohen Werte sorgen. Bei den kleineren Parteien FDP und Grünen, also den Parteien nahezu ohne Wahlkreismandate, sind die Quoten etwas geringer, liegen aber immer noch deutlich über 50 Prozent. So haben bei der SPD 84,1 Prozent aller Abgeordneten nie erfolglos vor dem ersten Einzug in den Bundestag kandidiert. Bei der CDU trifft das auf 81,3 Prozent und bei der CSU auf 83,3 Prozent zu. Unter den FDP-Abgeordneten ziehen 64,6 Prozent und unter den Grünen 68,4 Prozent mit ihrer ersten Kandidatur in den Bundestag ein. Eine interessante Ausnahme unter den kleinen Parteien bildet die PDS / Linkspartei. Von ihren Abgeordneten haben 81,2 Prozent nie erfolglos vor ihrem ersten Einzug in den Bundestag kandidiert.

Betrachtet man die erfolglosen Kandidaturen von Abgeordneten nach ihrem ersten Einzug in den Bundestag, stellt man fest, dass sich hier die PDS / Linkspartei auf demselben Niveau wie FDP und Grüne befindet. Bei der SPD haben nach dem ersten Mandat 82,6 Prozent nie erfolglos kandidiert, bei der CDU 79,9 und bei der CSU sogar 84,2 Prozent. Bei der FDP sinkt dieser Anteil auf 64,6 Prozent, bei den Grünen auf 65,1 und bei der PDS / Linkspartei auf 67,1 Prozent. Die Zahlen belegen eindrücklich den sehr hohen Stellenwert der Nominierung für die Wahl- und Wiederwahlchancen der Bewerber um ein Bundestagsmandat.

Ein zusammenfassender Überblick über die typischen politischen Karriereverläufe lässt sich mithilfe der Sequenzanalyse gewinnen. ${ }^{12}$ Mithilfe des neu entwickelten Sequenz-Ana-

11 Im Anhang findet sich die Beschreibung der Datenquellen. Dort wird auch der Prozess der Datengewinnung und -aufbereitung geschildert.

12 Vgl. Josef Brüderl / Stefani Scherer, Methoden zur Analyse von Sequenzdaten, in: Kölner Zeitschrift für Soziologie und Sozialpsychologie, 56. Jg. (2006), H. 44, S. 330 - 347; dies., Sequenzdatenanalyse, in: Christof Wolf I Henning Best (Hrsg.), Handbuch der sozialwissenschaftlichen Datenanalyse, Wiesbaden 2010, S. 1031 - 1051; Christian Brzinsky-Fay / Ulrich Kohler, New Develop- 


\begin{tabular}{|c|c|c|c|}
\hline \multicolumn{4}{|c|}{ Tabelle 1: Sequenzhäufigkeiten, Wahlbewerber und Bundestagsabgeordnete, 1949 bis 2009} \\
\hline Sequenz & $\mathrm{N}$ & Prozent & kumulierte Prozent \\
\hline 2 & 13.096 & 42.31 & 42.31 \\
\hline 1 & 6.321 & 20.42 & 62.73 \\
\hline 3 & 5.580 & 18.03 & 80.75 \\
\hline $2-2$ & 1.806 & 5.83 & 86.59 \\
\hline $3-3$ & 704 & 2.27 & 88.86 \\
\hline $1-1$ & 521 & 1.68 & 90.54 \\
\hline $2-2-2$ & 403 & 1.30 & 91.85 \\
\hline $3-2$ & 358 & 1.16 & 93.00 \\
\hline $2-3$ & 281 & 0.91 & 93.91 \\
\hline $1-3$ & 278 & 0.90 & 94.81 \\
\hline $3-1$ & 246 & 0.79 & 95.60 \\
\hline 7 & 221 & 0.71 & 96.32 \\
\hline $1-2$ & 208 & 0.67 & 96.99 \\
\hline $2-1$ & 197 & 0.64 & 97.63 \\
\hline 5 & 183 & 0.59 & 98.22 \\
\hline $3-3-3$ & 135 & 0.44 & 98.65 \\
\hline 6 & 113 & 0.37 & 99.02 \\
\hline $7-7$ & 110 & 0.36 & 99.37 \\
\hline $2-2-2-2$ & 105 & 0.34 & 99.71 \\
\hline 7-7-7 & 89 & 0.29 & 100 \\
\hline Gesamt & 30.955 & 100 & \\
\hline \multicolumn{4}{|c|}{$\begin{array}{l}\text { Legende: Wahlkreiskandidatur - 1, Listenkandidatur - 2, Doppelkandidatur - 3, Direktmandat nach } \\
\text { Wahlkreiskandidatur - 4, Listenmandat nach Listenkandidatur - 5, Direktmandat nach Doppelkandi- } \\
\text { datur - 6, Listenmandat nach Doppelkandidatur - 7. Lesebeispiel für Sequenz 3-2: erst eine Doppel-, } \\
\text { danach eine Listenkandidatur. } \\
\text { Quelle: Eigene Berechnungen. }\end{array}$} \\
\hline
\end{tabular}

lyse Moduls von Stata ${ }^{13}$ wurden sieben verschiedene Karriere-Episoden kodiert und ihre typischen Abfolgen, also Sequenzen, betrachtet. Die möglichen Episoden sind: (erfolglose) Wahlkreiskandidatur (1); (erfolglose) Listenkandidatur (2); (erfolglose) Doppelkandidatur (3); Direktmandat nach Wahlkreiskandidatur (4); Listenmandat nach Listenkandidatur (5); Doppelkandidatur mit erfolgreicher Wahl im Wahlkreis (6); Doppelkandidatur mit Einzug über die Liste (7). Aus Tabelle 1 sind die zwanzig häufigsten Sequenzen für die insgesamt 35.211 Personen zu entnehmen, die in den bislang siebzehn Bundestagswahlen um einen Sitz im Bundestag konkurriert haben. Diese zwanzig häufigsten Sequenzen betreffen knapp

ments in Sequence Analysis, in: Sociological Methods and Research, 38. Jg. (2010), H. 3, S. 359 -364 .

13 Vgl. Christian Brzinsky-Fay / Ulrich Kohler / Magdalena Luniak, Sequence Analysis with Stata, in: The Stata Journal, 6. Jg. (2006), H. 4, S. 435 - 460; dies., SQ: Stata Module for Sequence Analysis, Boston Department of Economics 2011. 


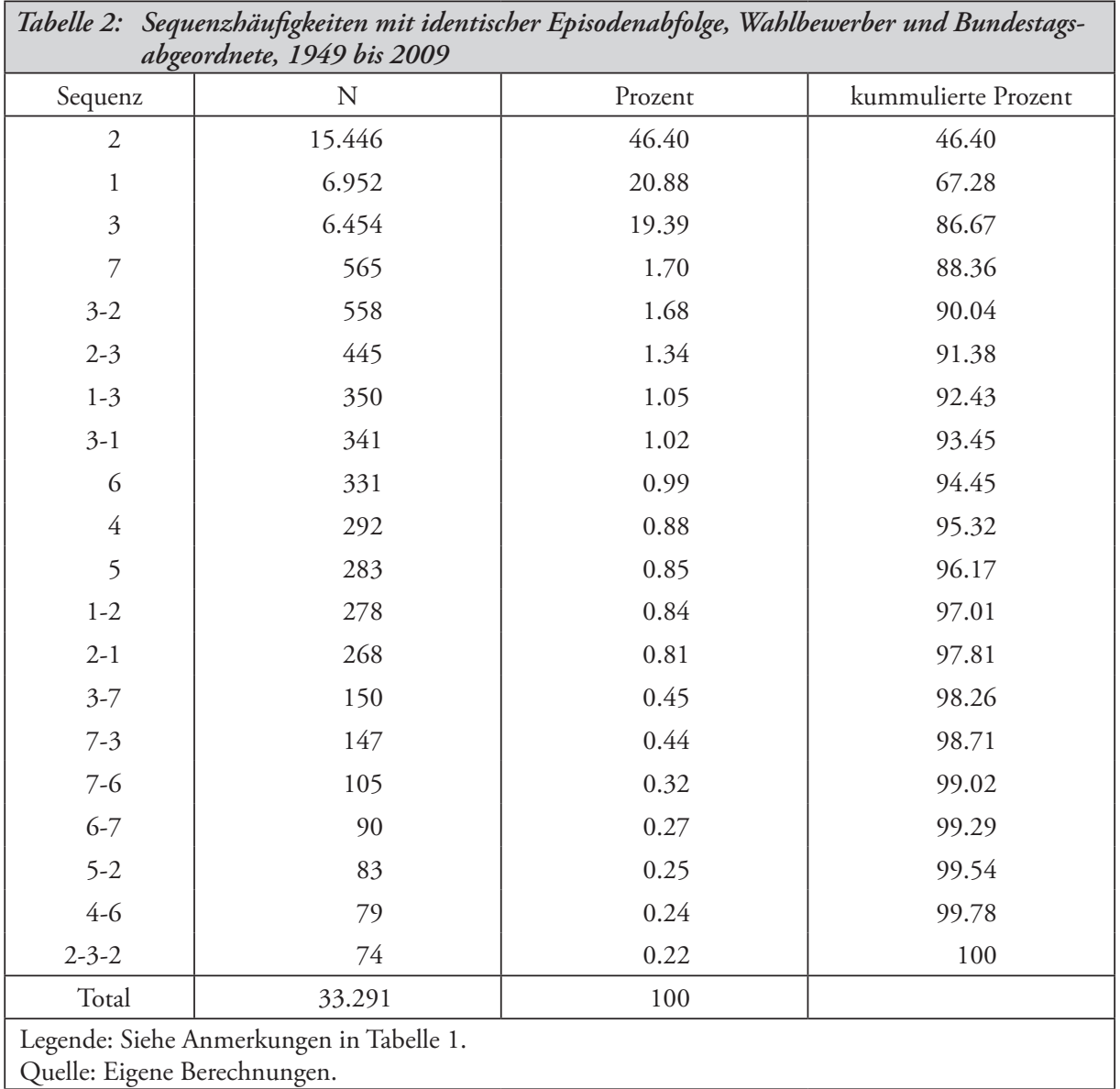

31.000 aller Kandidaten. Von diesen stellen allein die elf häufigsten Sequenzen erfolglose, ein-, zwei- oder auch dreimalige Kandidaturen dar. Unter den erfolgreichen Kandidaturen dominieren die reinen Listenkandidaten (5) und die Doppelkandidaten (6 und 7).

Beschränkt man die Betrachtung auf die Reihenfolge, in der die Episoden auftreten (fasst also etwa eine 3-3-7er und eine 3-7er Sequenz zusammen, genauso wie eine 6er und eine 6-6-6er Sequenz), so ergibt sich das in Tabelle 2 berichtete Bild. Nun geraten auch Sequenzen aus erfolglosen und erfolgreichen Kandidaturen $(3-7,7-3,5-2)$ in den Blick. Sie machen jedoch unter den hier betrachteten Sequenzen der etwa 33.300 Wahlbewerber nur circa ein Prozent $(\mathrm{N}=380)$ aus, berechnet auf die insgesamt 3.581 Abgeordneten etwas mehr als zehn Prozent.

Diese Befunde - überraschend in ihrer Eindeutigkeit - weisen dem Wähler also nur eine nachrangige Rolle in der Aus- und Abwahl der Bundestagsabgeordneten zu. Sie belegen das Wirksamwerden des grundlegendsten Disziplinierungsmechanismus der parlamentarischen Demokratie, über den Parteien gegenüber den Abgeordneten verfügen, die durch sie in die parlamentarische Verantwortung gekommenen sind - die Drohung mit der Nicht-Wiedernominierung. Weil eine sehr weitgehende Entscheidung über Wahl und Wiederwahl bereits mit der Nominierung getroffen ist, wird die Partei zum essentiellen Referenzpunkt in den 
Karrierekalkülen eines Jeden, der die Politik für sich zum Beruf gemacht hat oder machen will. Die Drohung mit der Nicht-Wiedernominierung ist eine effektive, glaubwürdige Drohung, weil sie tatsächlich in der Mehrzahl der Fälle das Ende der politischen Karriere bedeuten würde. Jedoch darf „Partei“ hier nicht als monolithischer Akteur und schon gar nicht als die Parteispitze verstanden werden. Die Einflusskonstellationen in den Nominierungsprozessen sind viel komplexer, als es das einfache Verständnis vom unbotmäßigen Abgeordneten, der von der Partei- und Fraktionsführung mit Nichtwiederaufstellung bedroht wird, suggeriert.

Zeigt sich somit die zentrale Bedeutung der Nominierung, so ist hinsichtlich der „deskriptiven Repräsentation“ zu vermuten, dass auch hier die Vorauswahl durch die Parteien entscheidender ist als die Auswahl durch die Wähler.

\section{Vor-Auswahl und Auswahl: Frauen als Kandidatinnen und Abgeordnete}

Obwohl die Wahl eines Abgeordneten nur die letzte Stufe in einem mehrstufigen Prozess der Selbst- und Fremdselektion ist, sind die der Wahl vorgelagerten Stufen wegen des Mangels an systematischen Daten zu Wahlbewerbern kaum untersucht worden. Grundlegende Fragen zu diesen vorgeschalteten politischen Selektionsmechanismen sind daher bislang ungeklärt, lassen sich aber mithilfe unseres Datensatzes nun beantworten. In diesem Kontext ist das bundesdeutsche gemischte Wahlsystem von besonderem Interesse, denn in der Literatur ist die Bedeutung der Wahlregeln für die parlamentarische Frauenrepräsentation un-

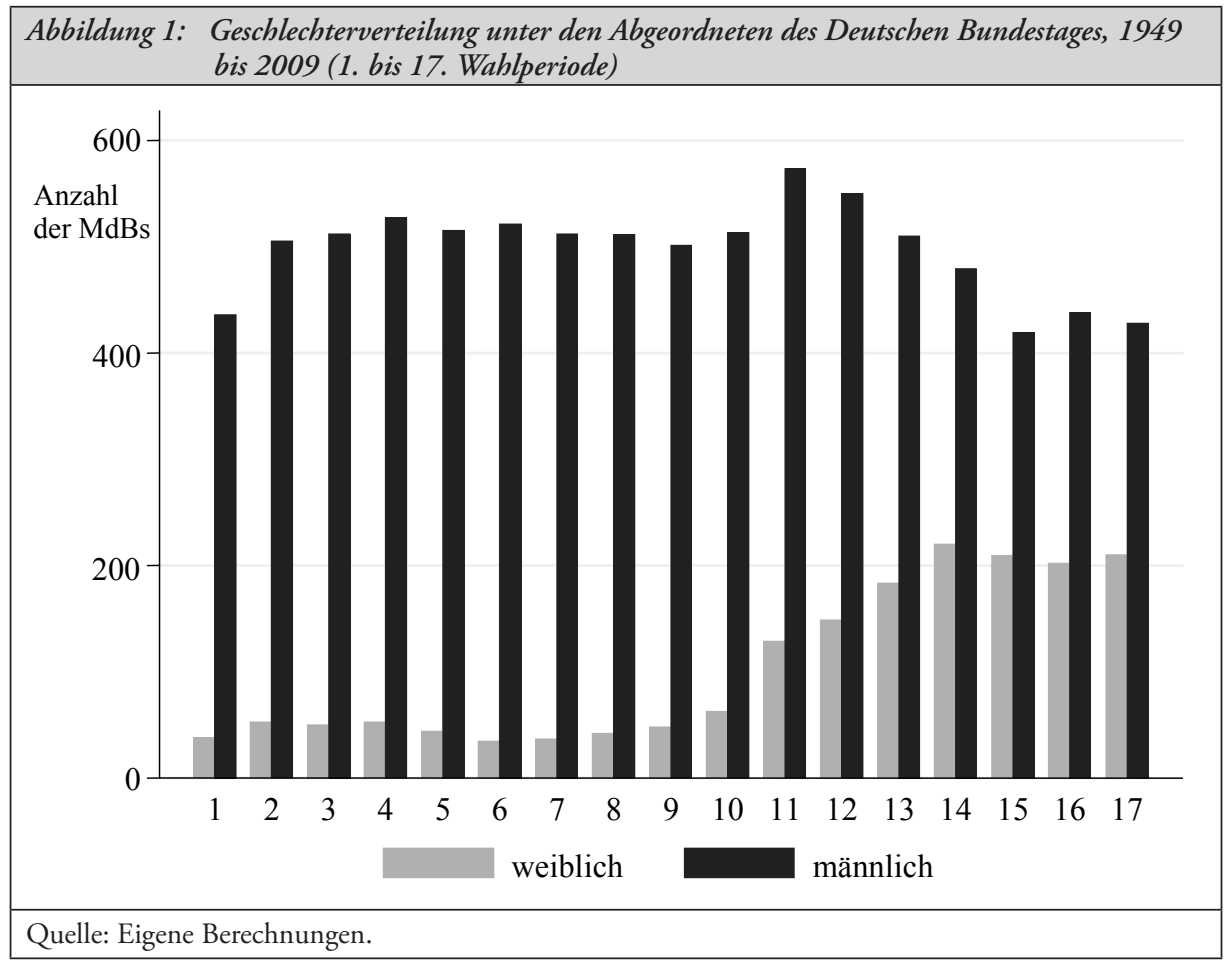




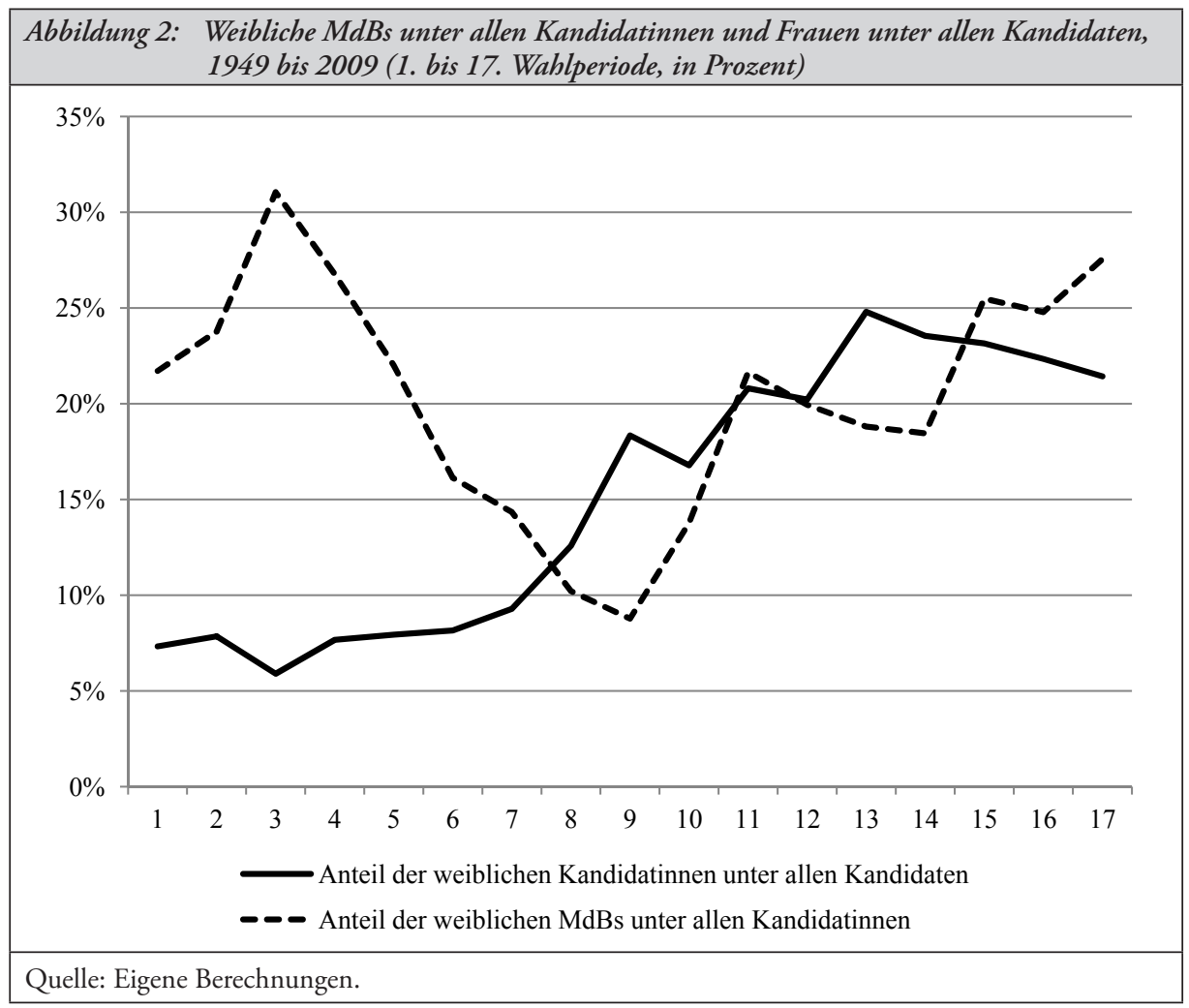

bestritten. ${ }^{14}$ Das deutsche gemischte Wahlsystem bietet dabei die Möglichkeit, die in der Literatur gut etablierte These zu überprüfen, die Verhältniswahl sei hinsichtlich der parlamentarischen Frauenrepräsentation vorteilhafter als die Mehrheitswahl. ${ }^{15}$ Eine Vielzahl anderer, wahrscheinlich ebenfalls einflussreicher Variablen kann dabei kontrolliert werden. Nach Parteien aufgegliederte Informationen zum Anteil der Kandidatinnen unter allen Wahlbewerbern versprechen zudem Aufschluss darüber, inwiefern der Zuwachs der Kandidatinnen ein Ansteckungseffekt ist, bei dem eine Partei eine Vorreiterrolle spielt, die von anderen Parteien dann schnell übernommen wird.

Ersichtlich wird der wachsende Frauenanteil sowohl unter den Wahlbewerbern als auch unter den Gewählten, wobei der Zuwachs an weiblichen Bundestagsabgeordneten seit der 14. Wahlperiode höher als der Zuwachs der Kandidatinnen ist. Dies ist als Hinweis darauf zu lesen, dass insbesondere die etablierten Parteien (a) einen überdurchschnittlichen Kandidatinnenanteil aufweisen und/oder (b) sie diese aussichtsreicher platzieren. Damit kommt die Frage in den Blick, wie genau Kandidatinnen eigentlich kandidieren: Auf welchen Listenplätzen sind Frauen zu finden? Die Abbildungen 3 und 4 zeigen die durchschnittliche relative Listenposition von Frauen und Männern für die SPD und CDU/CSU. Diese ergibt

14 Vgl. Richard E. Matland, Enhancing Women's Political Participation: Legislative Recruitment and Electoral Systems, in: IDEA (Hrsg.), Women in Parliament: Beyond Numbers, Stockholm 1998.

15 Vgl. Lena Wängnerud, a.a.O. (Fn. 9). 
sich, wenn man den individuellen Rangplatz durch die Gesamtzahl der Positionen der jeweiligen Landesliste teilt und dann Durchschnitte für weibliche und männliche Wahlbewerber bildet. Bei einer vollkommen „geschlechtsneutralen"Vergabe der Listenpositionen müssten die relativen Listenpositionen für Kandidaten und Kandidatinnen ununterscheidbar um 0.5 schwanken. Der Vergleich zwischen SPD und CDU/CSU zeigt jedoch durchaus ausgeprägte Unterschiede zwischen den Listenplatzierungen von Frauen und Männern und damit auch zwischen den Nominierungsstrategien der Parteien: Der deutliche zahlenmäßige Zuwachs der Kandidatinnen ist bei der SPD mit ihren im Vergleich zu den männlichen Mitbewerbern durchweg besseren Listenplatzierungen, bei der CDU/CSU mit ihren systematisch schlechteren Listenplatzierungen verbunden. Bei der Union ist dies seit der 8. Wahlperiode ein recht stabiles Muster, während bei der SPD Frauen seit der 12. Wahlperiode zunehmend bessere Chancen als Männer besitzen, über die Landesliste in den Bundestag einzuziehen.

Bei der Zahl weiblicher Wahlbewerber nehmen Grüne und SPD seit der 9. Wahlperiode (Abbildung 5), eine Vorreiterrolle ein. Ausgehend von einem nahezu identischen Frauenanteil unter den Bewerbern der etablierten Parteien von SPD, CDU/CSU und FDP um etwa 15 Prozent in der Wahl zum 9. Bundestag 1980 folgen die Parteien danach recht unterschiedlichen Pfadverläufen, wobei die FDP als die „männlichste“ Partei erscheint und im Zeitverlauf auch kaum eine Steigerung ihrer weiblichen Wahlbewerber verzeichnet.

Das deutsche gemischte Wahlsystem bietet die Möglichkeit zu drei unterschiedlichen Kandidaturformen: zur Wahlkreis-, Listen- oder Doppelkandidatur. Die Betrachtung der Ver-

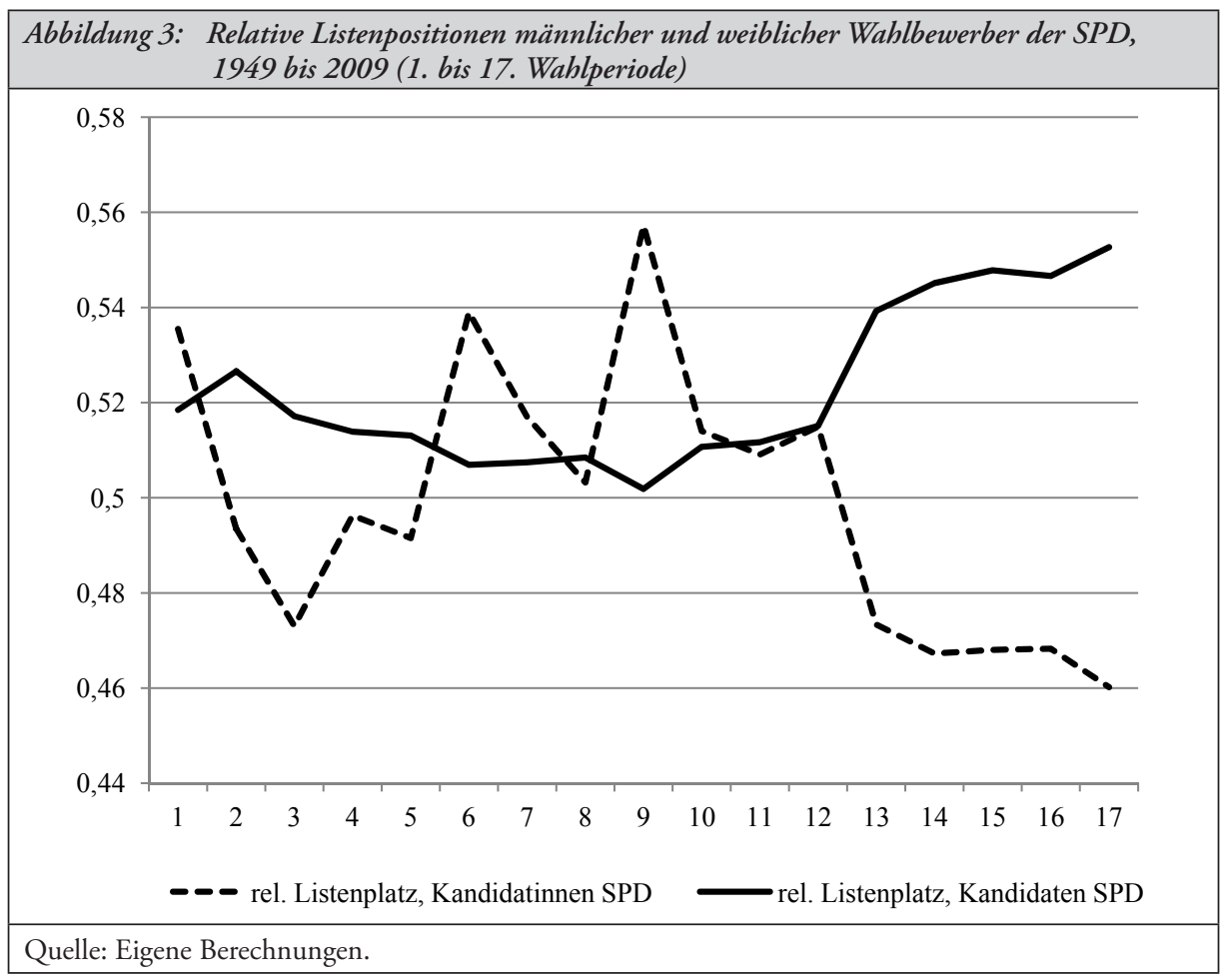



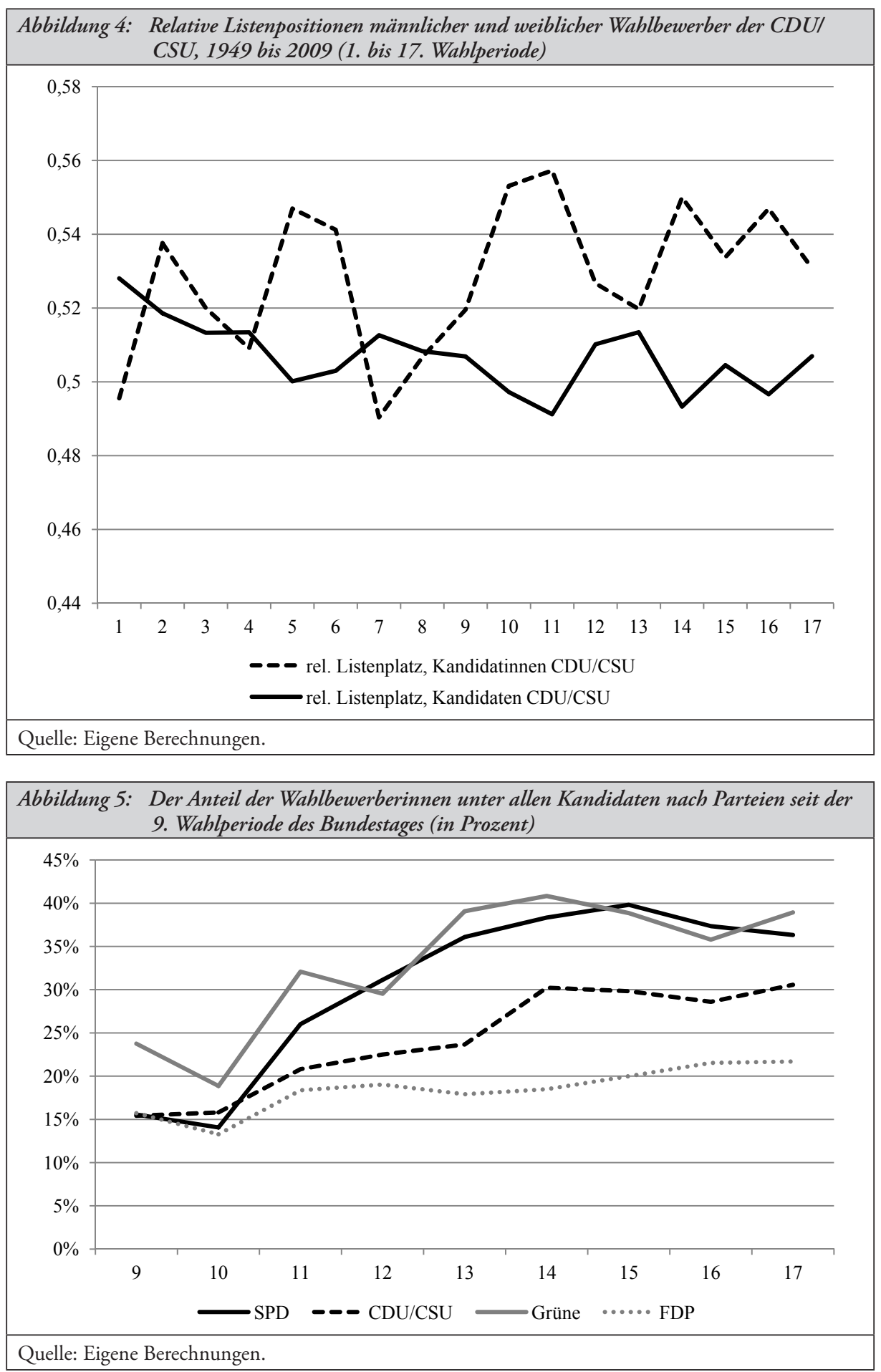


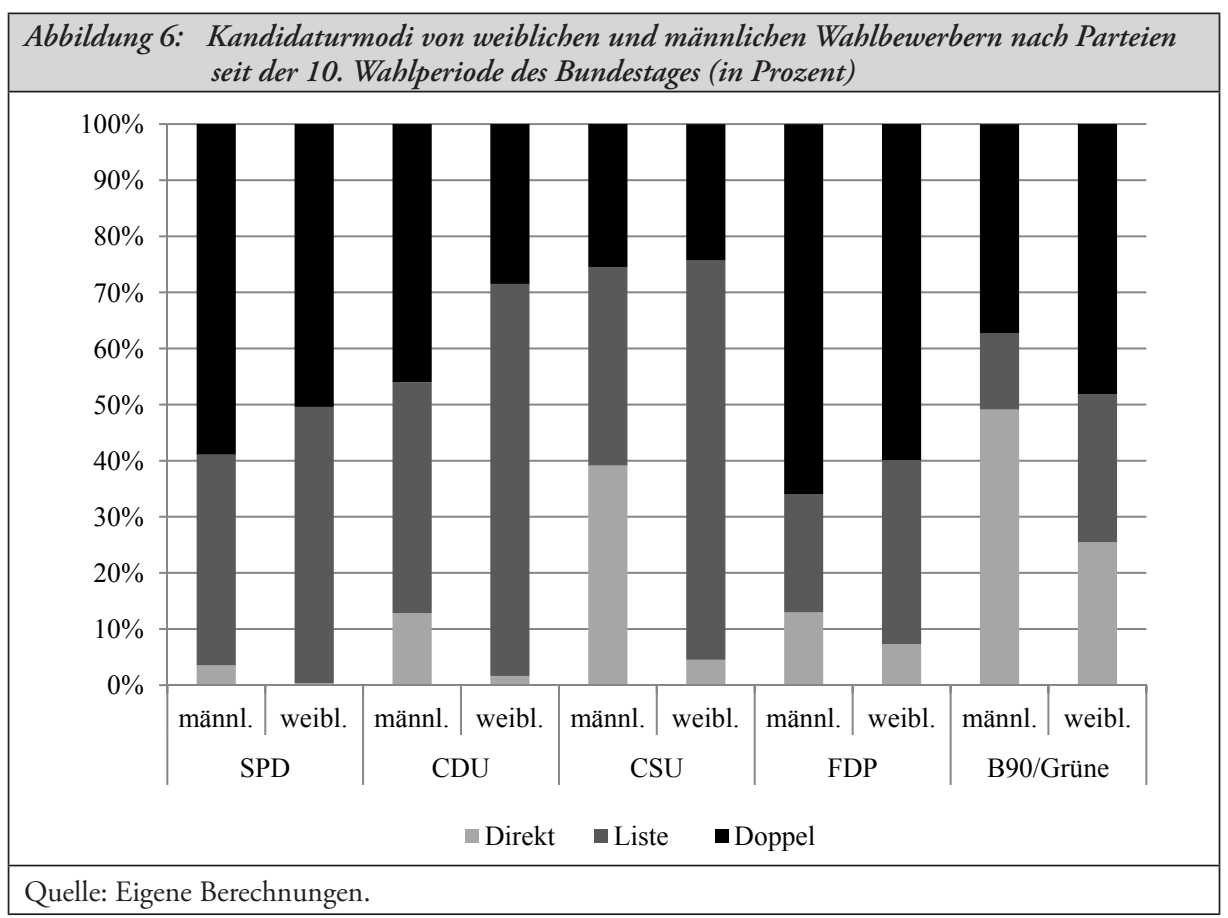

teilung dieser Kandidaturformen unter männlichen und weiblichen Wahlbewerbern - beschränkt auf die etablierten Parteien seit der 10. Wahlperiode - bestätigt den Befund der Literatur, dass die Verhältniswahl die parlamentarische Repräsentation von Frauen befördert. Unter den Wahlkreiskandidaten finden sich kaum Frauen - nur die Grünen stellten eine nennenswerte Zahl von Wahlkreiskandidatinnen auf (Abbildung 6).

Erneut erlaubt die Sequenzanalyse, typische Unterschiede in den Karriereverläufen weiblicher und männlicher Wahlbewerber zu ermitteln. Wir nutzen dazu eine neue Darstellungsform, den Sequence Modal Plot - als Darstellung einer „idealtypischen“ Sequenz (die in dieser Form nicht auch wirklich in dem Datensatz vorzukommen braucht). ${ }^{16}$ Die Identifizierung „ähnlicher“ Sequenzen erfolgt hierbei über die Levenshtein-Distanzen zwischen unterschiedlichen Sequenzen. ${ }^{17}$ Diese werden berechnet mithilfe der Zuordnung bestimmter Einfüge-, Lösch- und Substitutionskosten und der Berechnung der Zahl einzelner Einfüge- oder Substitutionsschritte, die notwendig sind, um eine Sequenz einer anderen vollständig anzugleichen. ${ }^{18}$ Abbildung 7 zeigt idealtypische Sequenzen getrennt nach Geschlecht ausgewiesen für alle Parteien und alle Bundestagswahlen seit 1949.

Auch in dieser Darstellungsform werden die Unterschiede im politischen Karriereverlauf von Männern und Frauen im politischen System der Bundesrepublik gut sichtbar. Die

16 Stata-Befehl: sqmodalplot, siehe Ulrich Kohler / Magdalena Luniak / Christian Brzinsky-Fay, SQ, a.a.O. (Fn. 13).

17 Vgl. dies., Sequence Analysis with Stata, a.a.O. (Fn. 13); Stefani Scherer/ Josef Brüderl, a.a.O. (Fn. 12).

18 Die default-Kostenzuweisung der Operationen „Insertion or Deletion“ beziehungsweise „Substitution" [indelcost(1) und subcost(2)] im Stata Sequenzanalyse-Modul wurde übernommen. 


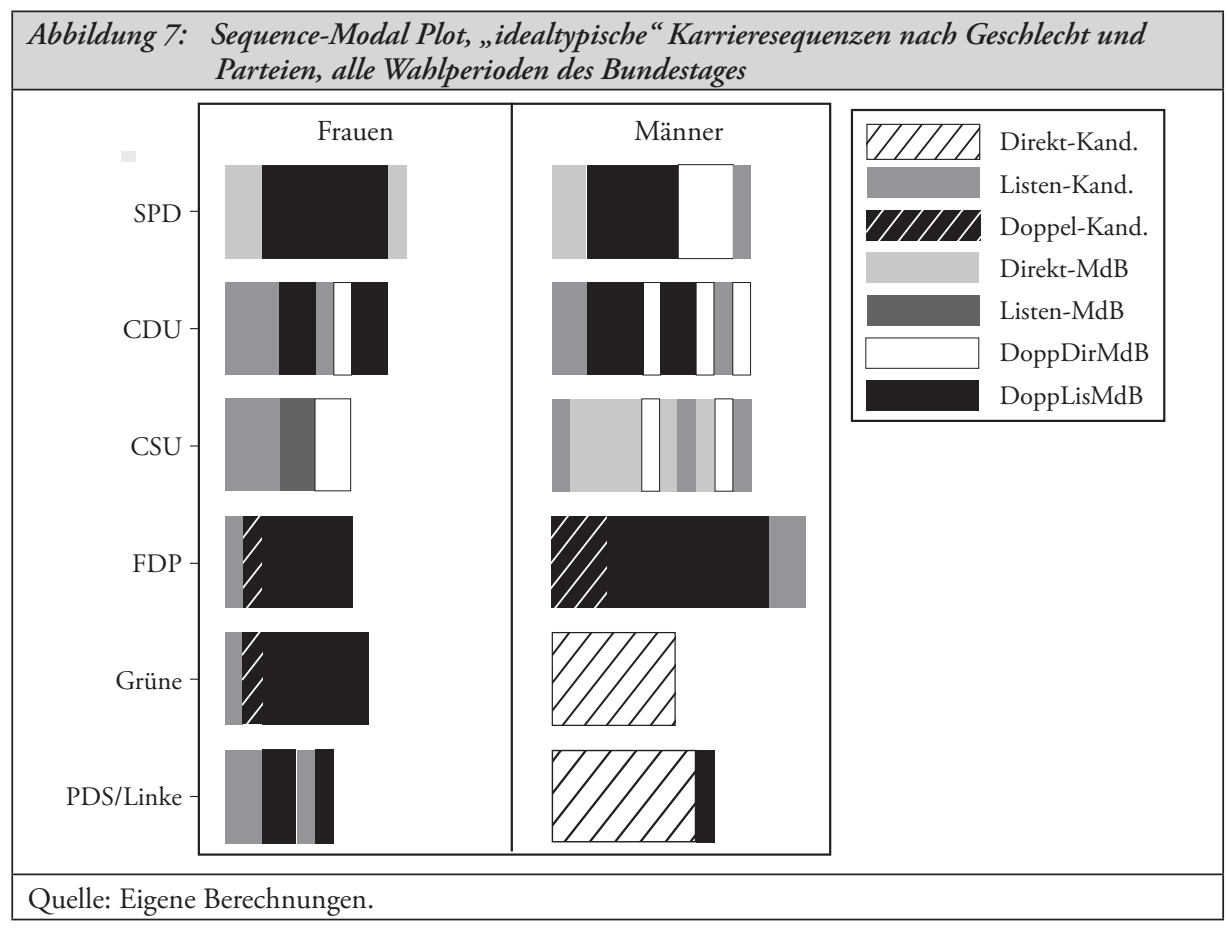

Dominanz entweder der (erfolglosen) Listenkandidatur oder der über die Liste erfolgreichen Doppelkandidatur unter den weiblichen Wahlbewerbern zeigt sich im Kontrast zu einer größeren Rolle des Direktmandats unter den männlichen Konkurrenten. Die erfolgreiche (reine) Direktkandidatur findet sich in dieser Rekonstruktion idealtypischer Karrieresequenzen allerdings nur bei der CSU. Die Unterschiede in den Karrieresequenzen (mit identischen Elementen) zwischen männlichen und weiblichen Bewerbern (ohne weitere Differenzierung nach Parteien) zeigt Tabelle 3.

Weibliche Bewerber sind demnach früher erfolgreich, und wenn sie es sind, dominiert eindeutig der Einzug über die Parteiliste. Unter den häufigsten 15 Sequenzen mit identischen Episoden kommen reine Wahlkreismandate (Sequenzelement 4) überhaupt nicht vor, reine erfolglose Wahlkreiskandidaturen sind deutlich seltener (um etwa zehn Prozent: 12,7 zu 22,5 Prozent) unter den Wahlbewerberinnen zu finden als unter ihren männlichen Konkurrenten.

\section{Wablen als Instrumente der Demokratie - ein Ausblick}

Mit dem vorliegenden Datensatz ist eine Vollerhebung aller jemals zu einer Bundestagswahl angetretenen Kandidaten verfügbar. Die Daten geben einen Komplettüberblick über wichtige Aspekte der nunmehr 60-jährigen Geschichte nationaler parlamentarischer Repräsentation in der Bundesrepublik Deutschland über. Sie bieten reichhaltige Informationen zu dem der Wahl vorgelagerten Prozess der Kandidatennominierung. Die damit ermöglichte Betrachtung des Zusammenwirkens von Vor-Auswahl durch die Parteien und Wahl durch 


\begin{tabular}{|r|rrr|r|rrr|}
\hline \multicolumn{7}{|c|}{ Tabelle 3: } & $\begin{array}{r}\text { Politische Karrieresequenzen weiblicher und männlicher Wablbewerber, alle Parteien, } \\
\text { alle Wahlperioden }\end{array}$ \\
\hline & \multicolumn{3}{|c|}{ Frauen } & \multicolumn{3}{c|}{ Männer } \\
\hline $\begin{array}{r}\text { Sequenz- } \\
\text { elemente }\end{array}$ & N & Prozent & $\begin{array}{c}\text { kumulierte } \\
\text { Prozent }\end{array}$ & $\begin{array}{r}\text { Sequenz- } \\
\text { elemente }\end{array}$ & N & Prozent & $\begin{array}{c}\text { kumulierte } \\
\text { Prozent }\end{array}$ \\
\hline $\mathbf{2}$ & 4.212 & 60.11 & 60.11 & $\mathbf{2}$ & 11.235 & 41.64 & 41.64 \\
$\mathbf{3}$ & 1.031 & 14.71 & 74.83 & $\mathbf{1}$ & 6.065 & 22.48 & 64.12 \\
$\mathbf{1}$ & 887 & 12.66 & 87.48 & $\mathbf{3}$ & 5.423 & 20.10 & 84.22 \\
$\mathbf{2 - 3}$ & 227 & 3.24 & 90.72 & $\mathbf{2 - 3}$ & 945 & 3.50 & 87.72 \\
$\mathbf{7}$ & 141 & 2.01 & 92.74 & $\mathbf{1 - 3}$ & 704 & 2.61 & 90.33 \\
$\mathbf{3 - 7}$ & 88 & 1.26 & 93.99 & $\mathbf{1 - 2}$ & 516 & 1.91 & 92.24 \\
$\mathbf{1 - 3}$ & 75 & 1.07 & 95.06 & $\mathbf{7}$ & 425 & 1.58 & 93.82 \\
$\mathbf{1 - 2}$ & 72 & 1.03 & 96.09 & $\mathbf{4}$ & 289 & 1.07 & 94.89 \\
$\mathbf{6}$ & 59 & 0.84 & 96.93 & $\mathbf{6}$ & 272 & 1.01 & 95.90 \\
$\mathbf{6 - 7}$ & 50 & 0.71 & 97.65 & $\mathbf{3 - 7}$ & 265 & 0.98 & 96.88 \\
$\mathbf{2 - 5}$ & 48 & 0.69 & 98.33 & $\mathbf{6 - 7}$ & 222 & 0.82 & 97.70 \\
$\mathbf{5}$ & 46 & 0.66 & 98.99 & $\mathbf{1 - 2 - 3}$ & 211 & 0.78 & 98.48 \\
$\mathbf{1 - 2 - 3}$ & 27 & 0.39 & 99.37 & $\mathbf{5}$ & 178 & 0.66 & 99.14 \\
$\mathbf{1 - 7}$ & 22 & 0.31 & 99.69 & $\mathbf{4 - 6}$ & 156 & 0.58 & 99.72 \\
$\mathbf{5 - 7}$ & 22 & 0.31 & 100 & $\mathbf{1 - 7}$ & 75 & 0.28 & 100 \\
\hline Total & 7.007 & 100 & & Total & 26.981 & 100 & \\
\hline Legende: Siehe Anmerkungen in Tabelle 1. & & & & \\
Quelle: Eigene Berechnungen. & & & & & \\
\hline
\end{tabular}

die Bürger liefert wichtige Antworten auf die Frage nach grundlegenden Mechanismen demokratischer Kontrolle und Sanktionierung. Der umfangreiche Datensatz erlaubt die Auswertung auch für kleine Parteien, ohne mit dem ernsthaften Problem zu niedriger Fallzahlen konfrontiert zu sein.

Zugleich besteht die grundsätzliche Möglichkeit, den Datensatz um weitere Variablen zu erweitern. Die Landeslisten sind deckungsgleich mit den Bundesländern. Damit können für jede beliebige Wahlperiode zusätzliche Informationen von der Landesebene aus externen Datensätzen zugespielt werden. Zu denken ist dabei zum Beispiel an Arbeitslosenquoten oder andere wirtschaftliche, soziale oder politische Indikatoren. Mit der Wahlkreisnummer liegt zudem ein kleinräumiger Indikator vor, der es erlaubt den Datensatz um differenzierte regionale Informationen zu ergänzen. Viele andere Erweiterungsmöglichkeiten sind denkbar. Aber auch ohne diese können nunmehr viele grundsätzliche Fragen nach der Effektivität des demokratischen Instruments der Wahl erstmals verlässlich auf einer umfassenden Datenbasis beantwortet werden. So werden zum Beispiel Kandidaturepisoden vor und nach der Mitgliedschaft im Bundestag - aufgegliedert nach Parteien, Zeitperioden und beispielsweise der Geschlechtsvariablen - sichtbar. In einem ersten Analyseschritt wurden diese hier untersucht.

Der Datensatz bietet zudem die Grundlage für weitere Analysen der Selektionseffekte im Wahlsystem. Hinsichtlich der deskriptiven Repräsentation von Frauen ist hier nicht nur der 
Vergleich von Kandidaturmodi instruktiv, sondern es kann auch ermittelt werden, inwiefern „Distriktgröße“ einen Einfluss auf die Repräsentation von Frauen im Parlament ausübt, denn nach vorherrschender Meinung ist der Wahlregeleffekt vor allem ein Distriktgrößeneffekt: Verhältniswahlsysteme mit ihren Mehrkandidatenwahlkreisen ermöglichen es den Parteien, ihre Listen nach verschiedenen Kriterien zu „balancieren“ - und ein Kriterium, das von Wahl zu Wahl an Bedeutung gewonnen hat, ist das Geschlecht. ${ }^{19}$ Je größer der Distrikt, desto mehr Balancierungsgelegenheiten, desto größer folglich die Auswahl, die dem Wähler geboten wird. Das deutsche Mischwahlsystem mit seinen unterschiedlich großen „secondary districts "20, also der unterschiedlichen Zahl der Mandate pro Land, ermöglicht eine direkte Überprüfung dieser Behauptung. Auch hierfür stellt der Kandidaten- und Abgeordneten-Datensatz die notwendigen Informationen zur Verfügung, um die genauen Auswahlmechanismen der repräsentativen Demokratie zu untersuchen.

\section{Anhang}

Der Kandidaten- und Abgeordnetendatensatz greift auf verschiedene Quellen zurück. Die Angaben zu den Bundestagskandidaten der Jahre 1949 bis einschließlich 1969 beruhen auf den Informationen der Kommission für Geschichte des Parlamentarismus und der politischen Parteien e.V., im Wesentlichen auf Martin Schumacher, und zwar auf der erweiterten Version der Online-Publikation. ${ }^{21}$ Erfasst sind hier sämtliche Kandidaten vom ersten bis zum sechsten Bundestag. Die Daten zu den Wahlbewerbern für den siebten Bundestag (1972) wurden mit Hilfe einer Texterkennungssoftware aus dem Sonderheft des Statistischen Bundesamtes extrahiert. ${ }^{22}$ Die Kandidatendaten für 1976 bis 2002 wurden vom Bundeswahlleiter zur Verfügung gestellt. Quelle der Angaben zu den Kandidaten der beiden letzten Bundestagswahlen (2005 und 2009) ist ebenfalls der Bundeswahlleiter, auf dessen Homepage neben den Wahlergebnissen auch vollständige Listen aller Kandidaten online verfügbar waren. ${ }^{23}$

Die Informationen über die Abgeordneten des Bundestages von 1949 bis zum Ende der 14. Wahlperiode (2002) sind ausschließlich dem Biographischen Handbuch der Mitglieder des Deutschen Bundestages entnommen. ${ }^{24}$ Die Ergebnisse der Wahlen von 2002, 2005 und 2009 und damit die im

19 Vgl. Richard E. Matland, a.a.O. (Fn. 14); ders. / Deborah Dwight Brown, District Magnitude's Effect on Female Representation in the U.S. State Legislatures, in: Legislative Studies Quarterly, 17. Jg. (1992), H. 4, S. 469 - 492; Annika Hennl / André Kaiser, Ticket-balancing in MixedMember Proportional Systems. Comparing Sub-national Elections in Germany, in: Electoral Studies, 27. Jg. (2008), H. 2, S. 321 - 336; dies., Wahlsysteme und Frauenrepräsentation. Ein Vergleich der deutschen Landesparlamente, in: ZPol, 18. Jg. (2008), H. 2, S. 167 - 184.

20 Gary W. Cox, Making Votes Count. Strategic Coordination in the World's Electoral Systems, New York 1997, S. 48 f.

21 Vgl. Martin Schumacher, M.d.B. - Die Volksvertretung 1949-1972, http://www.kgparl.de/onlinevolksvertretung/online-mdb.html (Abruf am 15. Juli 2011); ders., a.a.O. (Fn. 10).

22 Vgl. Statistisches Bundesamt, Die Wahlwerber für die Wahl zum 7. Deutschen Bundestag, Wiesbaden 1972.

23 Vgl. Der Bundeswahlleiter, http://www.bundeswahlleiter.de (Abruf am 15. Juli 2011).

24 Vgl. RudolfVierhaus / Ludolf Herbst, a.a.O. (Fn. 10), S. 4. 
Datensatz enthaltenen Informationen, ob bestimmte Kandidaten auch gewählt wurden, stammen wiederum von der Homepage des Bundeswahlleiters. Die Angaben über ausgeschiedene und nachgerückte Bundestagsabgeordnete dieser Zeitspanne wurden direkt den Internetseiten des Deutschen Bundestages entnommen. ${ }^{25}$ Dies ist auch die Quelle für die Informationen über Ausschussmitgliedschaften der Bundestagsabgeordneten von 2002 bis einschließlich 2011. Für den Zeitraum vor 2002 sind diese Informationen im Biographischen Handbuch berichtet.

Sämtliche Datenaufbereitungen wurden mit der Statistiksoftware Stata durchgeführt. Dazu wurden zunächst die einzelnen Wahlperioden separat aufbereitet, sodann die Einzeldatensätze zusammengeführt. Die Geschlechtsvariable wurde mit Hilfe von Vornamenstabellen, die mit den Vornamen im Datensatz verglichen wurden, generiert. Bei unklaren sowie äußerst seltenen Vornamen wurde der Geschlechter-Dummy manuell vergeben.

Die eindeutige Identifikation der Kandidaten ist die zentrale Voraussetzung für alle bisher genannten Forschungsfragen. Genau in dieser Erzeugung einer für identische Personen einheitlichen Identifikationsvariablen lag die größte Schwierigkeit. Während für die ersten sechs Bundestagswahlen IDWerte dank der Struktur der Datenquelle einfach zu erstellen waren ${ }^{26}$, gestaltete sich die Vergabe von IDs für die Kandidaten der restlichen elf Bundestagswahlen deutlich anspruchsvoller, denn es waren für jede Wahl nur separate Quellen verfügbar. Hinzu kommt, dass es immer wieder Abweichungen in der Schreibweise von Nach- und Vornamen wie auch in der Anzahl an aufgeführten Vornamen gibt. Außerdem können sich durch Heirat die Nachnamen der Kandidaten ändern. Mit Hilfe der Software „Merge-Tool-Box“27, die die Möglichkeit eines probabilistischen „Record Linkage“ bietet, konnten dennoch einheitliche IDs erzeugt werden. Dazu wurde ein Gesamtdatensatz der ersten sechs Bundestagswahlen erstellt, der jeden Kandidaten und damit jeden bisher vergebenen ID-Wert nur einmal enthielt. Alle Vor- und Nachnamen wurden für alle Bundestagswahlen in einzelnen Variablen gespeichert, genauso wie das Geburtsjahr. Danach wurde der Datensatz für die ersten sechs Wahlen mit dem Datensatz der Kandidaten der siebten Bundestagswahl mit Hilfe der MTB zusammengeführt. Je nach Wahrscheinlichkeit der Übereinstimmung der einzelnen Namenspaare und des Geburtsjahrs wurden durch die MTB (zuvor definierte) Werte vergeben, die eine Kategorisierung in „matched“, „probably matched“ und „not matched“ möglich machten. Die identifizierten Paare wurden danach zusätzlich einzeln überprüft. Jedem überprüften Personenpaar wurde sodann die ID zugewiesen, die die entsprechende Person bereits vor 1972 hatte. Neue Kandidaten bekamen eine neue ID. Im nächsten Schritt wurden die neuen Personen in den Gesamtdatensatz integriert und dieser wiederum mit dem Datensatz für die Kandidaten der achten Bundestagswahl zusammengeführt. Dieses Verfahren wurde für alle weiteren Bundestagswahlen wiederholt. Es konnten identische Personen trotz unterschiedlicher Schreibweisen oder sonstiger geringer Abweichungen bei Namen und Geburtsjahr abgeglichen werden. Bei der Einzelüberprüfung gelang es zudem, einige Personen zu identifizieren, die durch Heirat ihren Nachnamen vollständig geändert hatten. Wahrscheinlich konnten jedoch nicht alle diese Fälle komplett erfasst werden. Es ist daher nicht auszuschließen, dass im Datensatz unter den Kandidaten noch einige wenige Personen enthalten sind, denen unterschiedliche IDs zugewiesen wurden, obwohl es sich um dieselbe Person handelt. Für die Bundestagsabgeordneten lag im Datensatz des Biographischen Handbuchs der Bundestagsmitglieder bereits eine ID-Variable vor, so dass hier ein Abgleich mit den

25 Vgl. Deutscher Bundestag, http://www.bundstag.de (Abruf am 15. Juli 2011).

26 Die pdf-Datei ist in ihrer ursprünglichen Fassung nach den Namen der Kandidaten sortiert und fasst für jeden Namen sämtliche Kandidaturen von 1949 bis einschließlich 1969 zusammen. Damit kann eine ID-Variable durch einfaches Durchzählen vergeben werden.

27 Tobias Bacheteler, Merge ToolBox: Linkage Module, Version 0.71. Getting Started, Programmhandbuch, Duisburg 2010; Rainer Schnell / ders. I Stefan Bender, A Toolbox for Record Linkage, in: Austrian Journal of Statistics, 33. Jg. (2004), H. 1-2, S. 125 - 133. 


\begin{tabular}{|c|c|c|c|l|c|}
\hline \multicolumn{7}{|c|}{ Tabelle A1: } & \multicolumn{6}{c|}{ Vergleich der Angaben zu den Kandidaturen in den deutschen Bundestagswablen seit } \\
\hline 1949 zwischen Bundeswahlleiter 2009 (Soll) und unserem Datensatz (Haben)
\end{tabular}

generierten IDs stattfinden konnte. ${ }^{28}$ Vor allem deshalb ist bei den MdBs nicht damit zu rechnen, dass dieselben Personen verschiedene IDs auf sich vereinen.

Der fertige Datensatz enthält alle Kandidaten der 17 Bundestagswahlen von 1949 bis 2009 - also ein Komplettüberblick über die personelle Dimension von 60 Jahren parlamentarischer Repräsentation in der Bundesrepublik Deutschland. Er umfasst 53.760 Kandidaturen (Personenjahre) und 35.211 verschiedene Kandidaten (Personen). Darunter sind 10.215 erfolgreiche Kandidaturen (Abgeordnetenpersonenjahre), die wiederum auf 3.581 verschiedenen Personen (Abgeordnete) beruhen. Von den insgesamt 35.211 Wahlbewerbern waren 79,5 Prozent männlich, von den insgesamt 3.581 Mitgliedern des Bundestags waren dies 81,0 Prozent. Der Datensatz enthält Angaben zu Namen, Geschlecht, der Parteizugehörigkeit, der Kandidatur (einschließlich Wahlkreisnummer und/oder Listenplatz), zum Wahlergebnis (bei Erringen des Direktmandats) und zu den Mitgliedschaften in den Bundestagsausschüssen sowie eine Reihe weiterer biographischer Informationen.

Die folgenden Tabellen geben einen Überblick über die Kandidaten und Abgeordneten der SPD, CDU/CSU, FDP, der Grünen, der PDS/Die Linke und der übrigen Parteien. Die Differenz aus der

28 Mit Hilfe dieses Datensatzes konnte die durchgeführte Vergabe der IDs validiert werden, und es zeigte sich eindeutig, dass nahezu keine Fehler in den selbstgenerierten IDs vorlagen. Auf dieser Grundlage ist die Güte der Vergabe der IDs als hoch einzuschätzen. 
Summe der Listen- und Wahlkreiskandidaturen und der Anzahl an verschiedenen Kandidaten ergibt für das jeweilige Wahljahr die Anzahl an Doppelkandidaturen. Beispielsweise wies die SPD 2009438 Listenkandidaturen und 299 Wahlkreiskandidaturen auf, also 737 Kandidaturen. Insgesamt waren dafür 457 verschiedene Wahlbewerber verantwortlich. Die Differenz beträgt 280. Damit waren von den 457 Kandidaten 280 Doppelkandidaten. ${ }^{29}$

Es stellt sich bei diesem umfangreichen, aus verschiedenen Quellen gewonnenen Datensatz die Frage nach der Datenqualität. Um in etwa die Güte der vorliegenden Daten abschätzen zu können, wurde daher die Anzahl der Kandidaten im Datensatz (Haben) mit der aggregierten Überblicksaufstellung des Bundeswahlleiters ${ }^{30}$ und seinen offiziellen Angaben zu den Kandidaturen seit 1949 (Soll) verglichen (vgl. Tabelle A1).

Der Datenvergleich weist insgesamt 34 Fehler nach, verursacht durch 34 fehlende Kandidaturen. Berechnet auf die 53.760 Kandidaturen insgesamt, ergibt sich eine Fehlerquote von 0,063 Prozent vorausgesetzt, die abweichenden Angaben beruhen ausschließlich auf Fehlern in unserer Kandidatenund Abgeordnetendatenbank. Man kann also von einer ausgesprochen verlässlichen Datenquelle reden.

\begin{tabular}{|c|c|c|c|c|c|c|c|c|}
\hline Tabelle A & Kan & lidaten una & $d$ Gewählte d & - $S P D$, & 1949 bis 20 & 009 & & \\
\hline SPD & & Kandidatu & & & & Gew: & ählte & \\
\hline Wahljahr & Liste & Wahlkreis & Kandidaten & Liste & Wahlkreis & Gesamt & Ausgeschieden & Nachgerückt \\
\hline 1949 & 276 & 254 & 402 & 50 & 104 & 154 & 15 & 18 \\
\hline 1953 & 383 & 240 & 422 & 131 & 45 & 176 & 12 & 14 \\
\hline 1957 & 419 & 242 & 441 & 153 & 46 & 199 & 18 & 18 \\
\hline 1961 & 429 & 247 & 443 & 139 & 91 & 230 & 27 & 27 \\
\hline 1965 & 478 & 247 & 494 & 143 & 94 & 237 & 21 & 20 \\
\hline 1969 & 494 & 248 & 512 & 123 & 127 & 250 & 13 & 13 \\
\hline 1972 & 616 & 248 & 637 & 106 & 152 & 258 & 16 & 16 \\
\hline 1976 & 639 & 248 & 660 & 125 & 113 & 238 & 14 & 14 \\
\hline 1980 & 471 & 248 & 495 & 110 & 127 & 237 & 9 & 9 \\
\hline 1983 & 456 & 248 & 469 & 145 & 68 & 213 & 11 & 11 \\
\hline 1987 & 463 & 248 & 514 & 159 & 79 & 238 & 12 & 45 \\
\hline 1990 & 531 & 328 & 546 & 162 & 90 & 252 & 13 & 13 \\
\hline 1994 & 526 & 327 & 532 & 158 & 103 & 261 & 9 & 9 \\
\hline 1998 & 543 & 328 & 553 & 94 & 212 & 306 & 11 & 8 \\
\hline 2002 & 516 & 299 & 525 & 87 & 171 & 258 & 9 & 7 \\
\hline 2005 & 479 & 299 & 490 & 83 & 145 & 228 & 6 & 6 \\
\hline 2009 & 438 & 299 & 457 & 86 & 64 & 150 & 4 & 4 \\
\hline
\end{tabular}

29 Überschüsse bei den Nachrückern für das Wahljahr 1949 ergeben sich durch die Erhöhung der Anzahl Berliner Abgeordneter 1952 sowie durch Wahlkreisnachwahlen, bei denen beispielsweise ausgeschiedene SPD-Abgeordnete auch durch FDP-Abgeordnete abgelöst werden konnten. Die Überschüsse 1953 resultieren aus den Saarländer Abgeordneten, die für das Wahljahr 1987 sind durch die DDR-Volkskammer-Abgeordneten, die 1990 in den Bundestag rückten, zu erklären. Schließlich können seit 1998 für ausgeschiedene Direktmandatsträger aus Bundesländern mit Überhangmandaten keine Listenkandidaten nachrücken.

30 Der Bundeswahlleiter, Wahl zum 17. Deutschen Bundestag am 27. September 2009, Wiesbaden 2009. 


\begin{tabular}{|c|ccc|ccc|cc|}
\hline \multicolumn{7}{|c|}{ Tabelle A2b: Kandidaten und Gewählte der CDU/CSU, 1949 bis 2009 } \\
\hline CDU/CSU & \multicolumn{3}{|c|}{ Kandidaturen } & \multicolumn{5}{c|}{ Gewählte } \\
\hline Wahljahr & Liste & Wahlkreis & Kandidaten & Liste & Wahlkreis & Gesamt & Ausgeschieden & Nachgerückt \\
\hline 1949 & 251 & 242 & 437 & 36 & 119 & 155 & 10 & 14 \\
1953 & 344 & 221 & 478 & 97 & 172 & 269 & 19 & 20 \\
1957 & 382 & 245 & 516 & 101 & 194 & 295 & 18 & 18 \\
1961 & 403 & 247 & 543 & 123 & 156 & 279 & 29 & 28 \\
1965 & 441 & 247 & 555 & 113 & 154 & 267 & 17 & 16 \\
1969 & 445 & 248 & 560 & 148 & 121 & 269 & 19 & 19 \\
1972 & 614 & 248 & 726 & 146 & 96 & 242 & 8 & 8 \\
1976 & 631 & 248 & 727 & 137 & 134 & 271 & 17 & 17 \\
1980 & 501 & 248 & 597 & 133 & 120 & 253 & 16 & 16 \\
1983 & 462 & 248 & 544 & 93 & 179 & 272 & 17 & 17 \\
1987 & 462 & 248 & 601 & 153 & 169 & 322 & 18 & 87 \\
1990 & 526 & 328 & 600 & 102 & 235 & 337 & 18 & 18 \\
1994 & 509 & 328 & 579 & 82 & 221 & 303 & 9 & 9 \\
1998 & 516 & 327 & 602 & 147 & 112 & 259 & 14 & 14 \\
2002 & 502 & 299 & 570 & 131 & 125 & 256 & 8 & 8 \\
2005 & 524 & 299 & 598 & 88 & 150 & 238 & 15 & 12 \\
2009 & 460 & 299 & 530 & 26 & 218 & 244 & 7 & 5 \\
\hline Quelle: Eigene Darstellung. & \multicolumn{7}{c}{} & \\
\hline
\end{tabular}

\begin{tabular}{|c|c|c|c|c|c|c|c|c|}
\hline \multicolumn{9}{|c|}{ Tabelle A2c: Kandidaten und Gewählte der FDP, 1949 bis 2009} \\
\hline \multirow{2}{*}{$\begin{array}{c}\text { FDP } \\
\text { Wahljahr }\end{array}$} & \multicolumn{3}{|c|}{ Kandidaturen } & \multicolumn{5}{|c|}{ Gewählte } \\
\hline & Liste & Wahlkreis & Kandidaten & Liste & Wahlkreis & Gesamt & Ausgeschieden & Nachgerückt \\
\hline 1949 & 238 & 251 & 374 & 54 & 13 & 67 & 11 & 14 \\
\hline 1953 & 267 & 223 & 340 & 49 & 14 & 63 & 7 & 10 \\
\hline 1957 & 276 & 247 & 344 & 48 & 1 & 49 & 5 & 5 \\
\hline 1961 & 291 & 245 & 346 & 71 & 0 & 71 & 4 & 4 \\
\hline 1965 & 265 & 247 & 336 & 55 & 0 & 55 & 5 & 5 \\
\hline 1969 & 283 & 246 & 333 & 37 & 0 & 37 & 6 & 6 \\
\hline 1972 & 329 & 248 & 375 & 49 & 0 & 49 & 7 & 7 \\
\hline 1976 & 334 & 247 & 388 & 44 & 0 & 44 & 4 & 4 \\
\hline 1980 & 327 & 248 & 369 & 59 & 0 & 59 & 5 & 5 \\
\hline 1983 & 319 & 248 & 354 & 36 & 0 & 36 & 1 & 1 \\
\hline 1987 & 296 & 248 & 332 & 59 & 0 & 59 & 2 & 11 \\
\hline 1990 & 389 & 328 & 431 & 82 & 1 & 83 & 4 & 4 \\
\hline 1994 & 306 & 325 & 374 & 48 & 0 & 48 & 1 & 1 \\
\hline 1998 & 334 & 327 & 411 & 45 & 0 & 45 & 2 & 2 \\
\hline 2002 & 368 & 299 & 420 & 55 & 0 & 55 & 8 & 8 \\
\hline 2005 & 369 & 299 & 404 & 63 & 0 & 63 & 2 & 2 \\
\hline 2009 & 346 & 299 & 378 & 95 & 0 & 95 & 2 & 2 \\
\hline
\end{tabular}




\begin{tabular}{|c|rcc|rcc|cc|}
\hline \multicolumn{7}{|c|}{ Tabelle A2d: Kandidaten und Gewählte der Grünen, 1949 bis 2009 } \\
\hline Grüne & \multicolumn{3}{|c|}{ Kandidaturen } & \multicolumn{5}{c|}{ Gewählte } \\
\hline Wahljahr & Liste & Wahlkreis & Kandidaten & Liste & Wahlkreis & Gesamt & Ausgeschieden & Nachgerückt \\
\hline 1949 & 0 & 0 & 0 & 0 & 0 & 0 & 0 & 0 \\
1953 & 0 & 0 & 0 & 0 & 0 & 0 & 0 & 0 \\
1957 & 0 & 0 & 0 & 0 & 0 & 0 & 0 & 0 \\
1961 & 0 & 0 & 0 & 0 & 0 & 0 & 0 & 0 \\
1965 & 0 & 0 & 0 & 0 & 0 & 0 & 0 & 0 \\
1969 & 0 & 0 & 0 & 0 & 0 & 0 & 0 & 0 \\
1972 & 0 & 0 & 0 & 0 & 0 & 0 & 0 & 0 \\
1976 & 0 & 0 & 0 & 0 & 0 & 0 & 0 & 0 \\
1980 & 166 & 229 & 303 & 0 & 0 & 0 & 0 & 0 \\
1983 & 204 & 244 & 318 & 53 & 0 & 53 & 27 & 25 \\
1987 & 144 & 247 & 293 & 55 & 0 & 55 & 6 & 13 \\
1990 & 204 & 311 & 386 & 8 & 0 & 8 & 0 & 0 \\
1994 & 203 & 309 & 366 & 50 & 0 & 50 & 1 & 1 \\
1998 & 264 & 325 & 409 & 50 & 0 & 50 & 3 & 3 \\
2002 & 221 & 297 & 368 & 56 & 1 & 57 & 2 & 2 \\
2005 & 238 & 297 & 366 & 55 & 1 & 56 & 5 & 5 \\
2009 & 198 & 296 & 339 & 70 & 1 & 71 & 3 & 3 \\
\hline Quelle: Eigene Darstellung. & & & & & & \\
\hline
\end{tabular}

\begin{tabular}{|c|rcc|ccc|cc|}
\hline \multicolumn{7}{|c|}{ Tabelle A2e: Kandidaten und Gewählte der PDS/Linke, 1949 bis 2009 } \\
\hline PDS/Linke & \multicolumn{3}{|c|}{ Kandidaturen } & \multicolumn{5}{c|}{ Gewählte } \\
\hline Wahljahr & Liste & Wahlkreis & Kandidaten & Liste & Wahlkreis & Gesamt & Ausgeschieden & Nachgerückt \\
\hline 1949 & 0 & 0 & 0 & 0 & 0 & 0 & 0 & 0 \\
1953 & 0 & 0 & 0 & 0 & 0 & 0 & 0 & 0 \\
1957 & 0 & 0 & 0 & 0 & 0 & 0 & 0 & 0 \\
1961 & 0 & 0 & 0 & 0 & 0 & 0 & 0 & 0 \\
1965 & 0 & 0 & 0 & 0 & 0 & 0 & 0 & 0 \\
1969 & 0 & 0 & 0 & 0 & 0 & 0 & 0 & 0 \\
1972 & 0 & 0 & 0 & 0 & 0 & 0 & 0 & 0 \\
1976 & 0 & 0 & 0 & 0 & 0 & 0 & 0 & 0 \\
1980 & 0 & 0 & 0 & 0 & 0 & 0 & 0 & 0 \\
1983 & 0 & 0 & 0 & 0 & 0 & 0 & 0 & 0 \\
1987 & 0 & 0 & 24 & 24 & 0 & 24 & 0 & 24 \\
1990 & 149 & 71 & 173 & 18 & 1 & 19 & 2 & 2 \\
1994 & 163 & 200 & 269 & 27 & 4 & 31 & 1 & 1 \\
1998 & 181 & 248 & 333 & 35 & 4 & 39 & 3 & 3 \\
2002 & 182 & 274 & 344 & 0 & 2 & 2 & 0 & 0 \\
2005 & 302 & 290 & 488 & 52 & 3 & 55 & 1 & 1 \\
2009 & 227 & 297 & 373 & 62 & 16 & 78 & 2 & 2 \\
\hline Quelle: Eigene Darstellung. & & & & & & \\
\hline
\end{tabular}




\begin{tabular}{|c|rcc|ccc|cc|}
\hline \multicolumn{7}{|c|}{ Tabelle A2f: Kandidaten und Gewählte der Übrigen, 1949 bis 2009 } \\
\hline Übrige & \multicolumn{3}{|c|}{ Kandidaturen } & \multicolumn{5}{c|}{ Gewählte } \\
\hline Wahljahr & Liste & Wahlkreis & Kandidaten & Liste & Wahlkreis & Gesamt & Ausgeschieden & Nachgerückt \\
\hline 1949 & 560 & 966 & 1.175 & 78 & 20 & 98 & 18 & 18 \\
1953 & 1.075 & 1.102 & 1.600 & 39 & 11 & 50 & 3 & 5 \\
1957 & 1.018 & 959 & 1.428 & 13 & 6 & 19 & 2 & 2 \\
1961 & 893 & 772 & 1.251 & 0 & 0 & 0 & 0 & 0 \\
1965 & 858 & 692 & 1.135 & 0 & 0 & 0 & 0 & 0 \\
1969 & 1.047 & 662 & 1.255 & 0 & 0 & 0 & 0 & 0 \\
1972 & 750 & 543 & 1.041 & 0 & 0 & 0 & 0 & 0 \\
1976 & 1.120 & 798 & 1.492 & 0 & 0 & 0 & 0 & 0 \\
1980 & 888 & 552 & 1.218 & 0 & 0 & 0 & 0 & 0 \\
1983 & 782 & 428 & 1.038 & 2 & 0 & 2 & 1 & 1 \\
1987 & 698 & 644 & 1.099 & 4 & 0 & 4 & 2 & 2 \\
1990 & 1.014 & 972 & 1.559 & 0 & 0 & 0 & 0 & 0 \\
1994 & 1.368 & 815 & 1.803 & 0 & 0 & 0 & 0 & 0 \\
1998 & 2.168 & 1.148 & 2.754 & 0 & 0 & 0 & 0 & 0 \\
2002 & 1.059 & 476 & 1.315 & 0 & 0 & 0 & 0 & 0 \\
2005 & 994 & 578 & 1.302 & 0 & 0 & 0 & 0 & 0 \\
2009 & 1.036 & 705 & 1.479 & 0 & 0 & 0 & 0 & 0 \\
\hline Quelle: Eigene Darstellung. & & & & & & \\
\hline
\end{tabular}

\title{
Der Wandel von Mitgliederparteien zu Wählerparteien - Setzt das Grundgesetz einen bestimmten Parteientypus voraus?
}

\author{
Kathrin Groh
}

Aufgeregtheiten um vermeintliche Krisen der Parteien sind so alt wie die Parteien selbst. Wer heute nicht von einer Krise der Parteiendemokratie redet, diagnostiziert mindestens einen Strukturwandel der deutschen Parteien. Korrespondiert dieser neue Typus Partei noch mit den Anforderungen, die das Grundgesetz an die Parteien stellt? Denn in Deutschland haben die Parteien eine ganz besondere verfassungsrechtliche Last zu tragen. Sie waren und sind gefangen zwischen (alt)liberalen Leitbildern, die von einer normativen Demokratietheorie überhöht werden, und der realen Entwicklung des deutschen Parteien-staates. Was aber, wenn die Realität der Parteiendemokratie von den verfassungsgerichtlich überformten Vorgaben des Grundgesetzes abzuweichen beginnt? Muss sich das Verfassungsrecht dann der Prägekraft der Parteiendynamik anpassen ${ }^{1}$ oder wird es -

1 Kritisch Michael Stolleis, Parteienstaatlichkeit - Krisensymptome des demokratischen Verfassungsstaats, in: VVDStRL, 44. Jg. (1986), S. 7 - 40, S. 13 f. 